\title{
MÉTODOS MULTIVARIADOS NA ANÁLISE DE ÓLEOS ESSENCIAIS DE Lippia origanoides Kunth CULTIVADA
}

\author{
$\underline{\text { Taiara Souza Costa }}{ }^{1}$; Maurício Santana Lordêlo ${ }^{2}$ \\ 1. Bolsista PROBIC/UEFS, Graduando em Agronomia, Universidade Estadual de Feira de \\ Santana, e-mail: \\ 2. Orientador, Departamento DEXA, Universidade Estadual de Feira de Santana, e-mail: \\ mslordelo@gmail.com
}

Palavras-chave: Compostos; Biorreguladores; Multivariada;

\section{INTRODUÇÃO}

A denominação "Análise Multivariada" corresponde a um grande número de métodos e técnicas que utilizam, simultaneamente, todas as variáveis na interpretação teórica do conjunto de dados obtidos Neto (2004). Logo, existem diferentes métodos na estatística multivariada, os quais cada método tem sua fundamentação teórica e sua aplicabilidade. Quando o interesse é verificar como as variáveis se relacionam, destacam-se dois métodos, que podem ser utilizados: análise de componentes principais e a análise de agrupamento hierárquico.

A análise de componentes principais (ACP) tem por objetivo descrever os dados contidos num quadro indivíduos-variáveis numéricos: $p$ variáveis serão medidas com $n$ indivíduos. Neste método, a redução do número de variáveis não se faz por uma simples seleção de algumas variáveis, mas pela construção de novas variáveis sintéticas, utilizando a informação contida na matriz de covariância dos dados ou matriz de correlação, princípios da álgebra linear e estatística básica (Bouroche \& Saprota, 1982).

$\mathrm{Na}$ análise de Agrupamento, pode-se agrupar amostras ou variáveis (pontos) de acordo com o interesse em cada situação. É possível calcular a distância de um ponto a todos os outros pontos, constituindo-se assim uma matriz que descreve a proximidade entre todas as amostras estudadas. Baseada nesta matriz de proximidade constrói-se um diagrama de similaridade denominado dendrograma Ferreira (2011).

A espécie alvo para o estudo foi Lippia origanoides Kunth Fígura 01 é arbusto aromático produz óleo essencial composto por carvacrol, timol, $\rho$-cimeno e $\gamma$-terpineno (Oliveira et al., 2007), com ação antimcrobiana (Oliveira et al., 2007), antiparasitária e antitumoral (Escobar et al., 2010). Visando aumentar a produtividade de culturas de plantas com metabólitos secundários de interesse comercial, como os óleos essenciais, metodologias que intensifiquem os processos fisiológicos ligados à síntese destes compostos vêm sendo avaliadas. Aplicações de hormônios e reguladores vegetais de crescimento (Silva et al., 2009); (Scavroni et al., 2006), bem como de substâncias sinalizadoras de ataque de patógenos e herbívoros em plantas (Deschamps \& Simon, 2006) podem ser utilizadas em culturas com esta finalidade, possibilitando assim um controle do seu metabolismo.

Este trabalho tem como objetivo analisar o efeito de pulverizações foliares de plantas cultivadas de Lippia origanoides Kunth com biorreguladores vegetais sobre o conteúdo e a composição de óleo essencial utilizando a análise de componente principal e análise de agrupamento por meio do software livre R.

MATERIAL E MÉTODOS

Os dados utilizados foram obtidos por meio do experimento, em que as unidades experimentais (plantas) receberam três pulverizações foliares, com intervalos de 14 dias, dos tratamentos: ácido giberélico (GA3), 6-benzilaminopurina (BAP), ácido acetilsalicílico 
(AAS), ácido jasmônico (AJ), Stimulate (STM) e água destilada (H20) que serviu como testemunha. Sete dias após a finalização da aplicação, três plantas foram coletadas de forma aleatória de cada tratamento. Folhas e inflorescências foram utilizadas para a obtenção do óleo essencial por hidrodestilação, tendo seu rendimento por planta e teor na matéria seca sido calculado. Os componentes químicos de cada repetição foram analisados com quantificação de 85 compostos, dos quais 22 foram identificados, correspondendo a 93,77\% do óleo essencial.

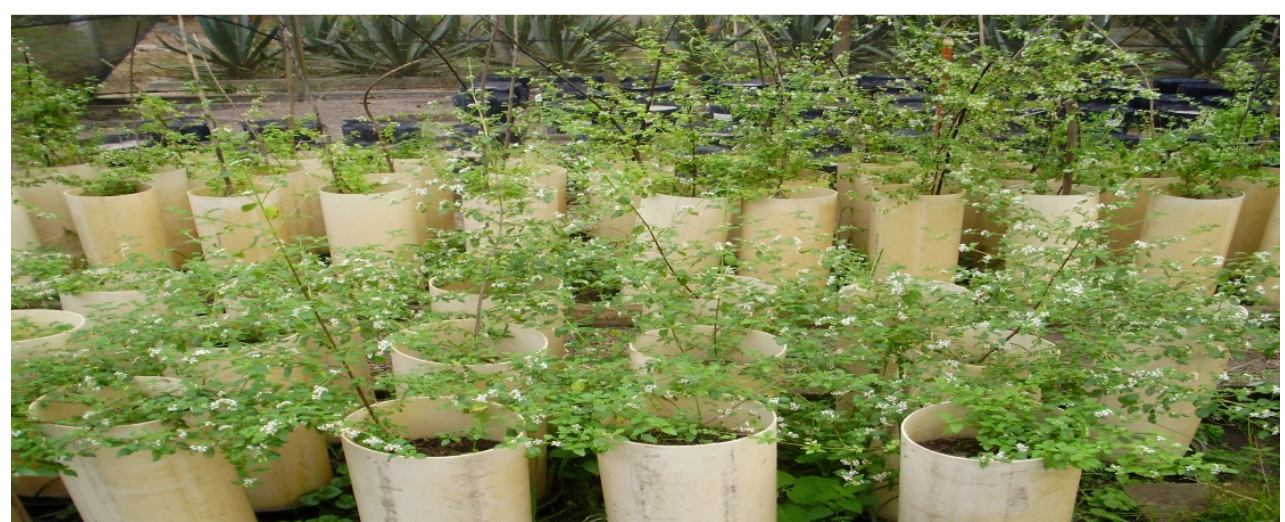

Fígura 1: Lippia origanoides Kunth

$\mathrm{Na}$ análise de componente principal, as variáveis $X 1, X 2, \ldots, X p$ medidas em cada um dos $n$ indivíduos ou unidades experimentais que origina uma matriz de dados $\mathbf{X}(n \times p)$ que possui uma matriz de covariância $\boldsymbol{\Sigma}$ e uma matriz de correlação $\mathbf{R}$, ambas $p \times p$. Associado a $\boldsymbol{\Sigma}$, obtém-se os pares de autovalores e autovetores $(\lambda 1, a 1),(\lambda 2, a 2), \ldots,(\lambda \mathrm{p}, a p)$ de modo que $(\lambda 1>\lambda 2>\ldots>\lambda p)$. O $i$-ésimo componente principal é definido por (Johnson \& Wichem, 1998):

$$
Z_{i}=a_{i 1} X_{1}+a_{i 2} X_{2}+\ldots+a_{i p} X_{p}
$$

A variância de $Z i$ é dada por:

$$
\operatorname{Var}\left(Z_{i}\right)=\operatorname{Var}\left(a_{i}^{\prime} X\right)=a_{i}^{\prime} \operatorname{Var}(X) a_{i}=a_{i}^{\prime} \sum a_{i}^{\prime}
$$

Por meio de procedimentos algébricos, pode-se mostrar que a variabilidade total contida nas variáveis originais é igual à variabilidade total contida nos componentes principais. A proporção da variância total da matriz $\mathbf{X}$ explicada pelo $i$-ésimo componente principal é dada por:

$$
\frac{\operatorname{Var}\left(Z_{i}\right)}{\sum_{i=1}^{p} \operatorname{Var}\left(Z_{i}\right)}=\frac{\lambda i}{\lambda 1+\lambda 2+\cdots+\lambda p}
$$

Pela proporção de explicação da variância total, que o modelo de $p$ componentes principais é responsável, pode-se determinar o numero de componentes que se deve reter (Johnson \& Wichem, 1998).

$\mathrm{Na}$ análise de agrupamentos, a similaridade entre duas amostras pode ser expressa como uma função da distância entre os dois pontos representativos destas amostras no espaço n-dimensional. A maneira mais usual de calcular a distância entre dois pontos a e b no espaço n-dimensional é conhecida por distância euclidiana (xab) e é dada por:

$$
X_{a \square}^{2}=\sum_{j=1}^{n}\left(d_{a j}-d_{\square j}\right)^{2}
$$

A técnica de agrupamento hierárquico interliga as amostras por suas associações, produzindo um dendrograma onde as amostras semelhantes, segundo as variáveis escolhidas, 
são agrupadas entre si. A suposição básica de sua interpretação é esta: quanto menor a distância entre os pontos, maior a semelhança entre as amostras (Neto et al., 1997).

Uma alternativa consiste em agrupar os pares de pontos que estão mais próximos, usando a distância euclidiana, e substituí-los por um novo ponto localizado na metade da distância entre eles. Este procedimento, quando repetido até que todos os pontos sejam agrupado em um só ponto, leva a construção do dendrograma, onde, no eixo horizontal são colocadas as amostras e, no eixo vertical, o índice de similaridade, sij, entre os pontos i e j, calculado segundo a seguinte expressão:

$$
S_{i j}=1-\frac{d_{i j}}{d_{\text {máx }}}
$$

onde dij é a distância entre os pontos i e j e dmáx é a distância máxima entre qualquer par de pontos (Neto et al., 1997). O dendrograma, portanto, consistem em diagramas que representam a similaridade entre pares de amostras através da formação de grupo os quais são diferentes entre si.

Todas as análises foram feitas por meio de rotinas computacionais implementadas no software $\mathrm{R}$ (R Core Team, 2015). A análise de Componente Principal foi realizada com o pacote "bpca", versão 1.2-2 (Faria et al., 2013).

\section{RESULTADO E DISCUSSÃO}

Com base nos resultados obtidos pela técnica dos componentes principais, as duas primeiras componentes principais foram responsáveis por $90.5 \%$ da variação total, sobre biorreguladores vegetais na produção dos compostos químicos presentes no óleo essencial de Lippia origanoides KUNTH, sendo que a componente principal 1 (CP1) foi responsável por $70.9 \%$ e o segunda CP2, por 19,6\% desta variação. A CP1 está representada expressivamente pelos compostos Timol e Carvacrol, estes dois sofreram maior influência dos tratamentos BAP e STM na primeira época considerada 15 dias. Já a CP2 está representado pelos compostos E_cariofileno e Biciclogermacreno, sendo que estes foram mais influenciados pelos tratamentos GA3 e STM na última época a qual foi de 45 dias e $\mathrm{H} 2 \mathrm{O}$ em todas as épocas de aplicação. O biplot, representado na Figura 2-A expressa esse resultado.

$\mathrm{Na}$ análise de agrupamento hierárquico com o método Ward, o dendrograma Figura 2$\mathrm{B}$ formou seis grupos entre os tratamentos que apresentam maior similaridade quanto à produção dos compostos químicos. Os grupos I e VI são formados pelos tratamentos que contribuíram para maiores produção dos compostos Timol e Carvacrol, sendo que o grupo I foi formado pelos tratamentos que mais contribuíram para a produção destes compostos. $\mathrm{O}$ grupo II é formado pelos tratamentos que contribuíram para maior produção de E-cariofileno e Biciclogermacreno, representantes da CP2.
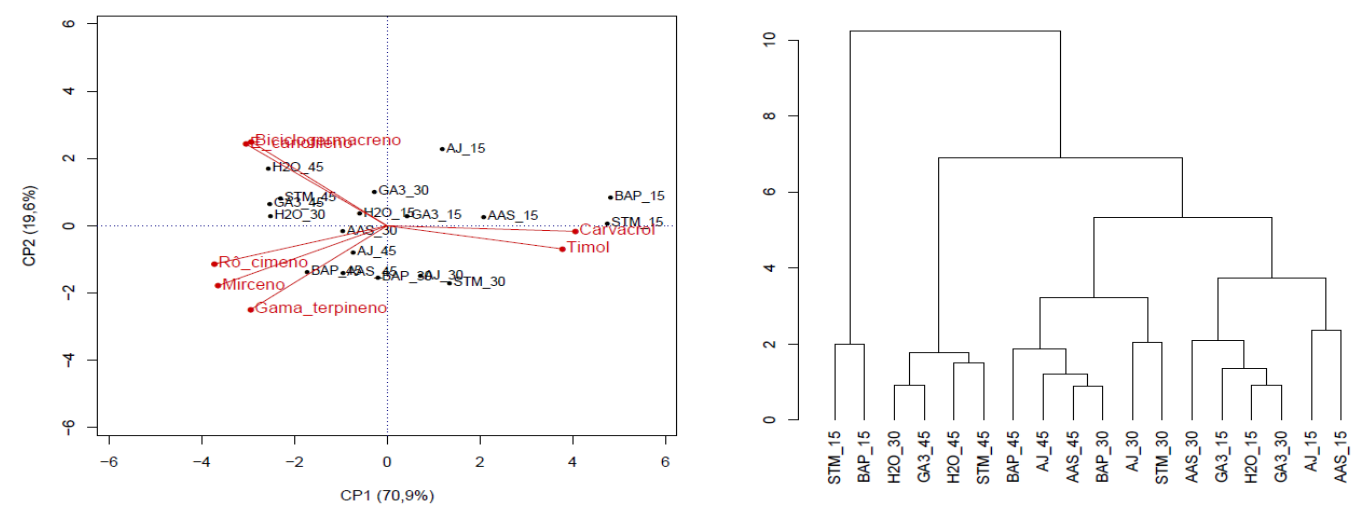

Figura2 A: Biplot CP1xCP2 com os indivíduos (tratamentos) sobre os compostos químicos. Figura2 B: Gráfico do Dendrograma pela análise de agrupamento - WARD. 


\section{CONCLUSÃO}

De acordo com os resultados, a análise de componentes principais permitiu a redução do número de variáveis (compostos) retirando as que apresentaram baixa variabilidade ou que foram redundantes por estarem correlacionadas com as de maior importância para dois componentes principais. Dessa forma, um menor número de compostos foi suficiente para explicar a variação total dos dados sem perda significativa de informação. Pela formação dos grupos entre os tratamentos, pode-se observar que não há um efeito isolado do tipo de biorregulador ou da época de aplicação sobre a síntese dos componentes químicos do óleo essencial de Lippia origanoides KUNTH, mas uma interação entre esses dois fatores, o que impede uma generalização do biorregulador e época de aplicação o que favorecem a síntese de um ou de outro composto.

\section{REFERENCIAS}

BOUROCHE, J. M.; SAPORTA, G. Análise de dados. Rio de Janeiro: Zahar, 1982.

DESCHAMPS, C. \& SIMON, J.E. Terpenoid essential oil metabolism in basil (Ocimum basilicum L.) following elicitation. The Journal of Essential Oil Research, v. 18, p. 618621, 2006.

ESCOBAR, P. et al. Chemical composition and antiprotozoal activities of Colombian Lippia spp. essential oils and their major components. Memorial Instituto Oswaldo Cruz, v. 105, n. 2, p. 184-190, 2010.

FARIA, José Cláudio; DEMÉTRIO, Clarice Garcia Borges; ALLAMAN, Bezerra. Biplot of Multivariate Data Based on Principal Components Analysis. 2013. Disponível em: $<$ https://cran.r-project.org/web/packages/bpca/bpca.pdf>. Acesso em: 06 out. 2017.

FERREIRA, D. F. Estatística Multivariada. 2a edição. Lavras: Ed. UFLA, 2011. JOHNSON, R.A.; WICHERN, D.W. Applied multivariate statistical analysis. Madison: Prentice Hall International, 1998. 816p.

MOITA NETO, José Machado.; MOITA, Graziella Ciaramella.Uma introdução à análise exploratória de dados multivariados, 1997.

NETO, M. M. J. Estatística multivariada. Revista de Filosofia e Ensino. 9 maio 2004.

OLIVEIRA, D.R. et al. Chemical and antimicrobial analyses of essential oil of Lippia origanoides H.B.K. Food Chemistry, v. 101, p. 236-240, 2007.

R CORE TEAM. R: a language and environment for statistical computing. Vienna: R Foundation for Statistical Computing. Disponível em: <http://www.r-project.org>, 2015.

SCAVRONI, J. et al. Rendimento e composição química do óleo essencial de Mentha piperita L. submetida a aplicações de giberelina e citocinina. Revista Brasileira de Plantas Medicinais, v. 8, n. 4, p. 40-43, 2006.

SILVA, M.M. et al. Variação de timol e carvacrol em Lippia microphylla Cham. ao longo do dia. In: 32 ${ }^{\mathbf{a}}$ Reunião Anual da Sociedade Brasileira de Química, Fortaleza - CE, 2009. 